\title{
Psicooncología
}

ISSN: $1696-7240$

\section{Desesperanza y su relación con el apoyo social percibido en pacientes peruanos con neoplasias hematológicas}

\author{
Juan Ricardo Peralta Gil ${ }^{1 *}$; Amy Mishelle Mariño Rugel²; Cristian Edwin Adriano Rengifo ${ }^{3}$
}

Recibido: 23 de abril de 2021 / Aceptado 30 de junio de 2021

Resumen: Objetivo: El propósito del presente estudio fue determinar la relación significativa entre el apoyo social y la desesperanza en pacientes peruanos con neoplasias hematológicas que se encuentren en alguna de las fases del tratamiento. Método: El estudio fue de tipo descriptivo-correlacional. La muestra estuvo conformada por 110 pacientes hematooncológicos de ambos sexos, con edades entre 16 a 25 años. Los instrumentos utilizados fueron la Escala Multidimensional de Apoyo Social Percibido (MSPSS) y la Escala de Desesperanza de Beck (BHS). Resultados: Se reportan niveles altos de apoyo social percibido ( $81 \%$ ) y niveles normales de desesperanza ( $82 \%)$ en los pacientes hematooncológicos. El apoyo social percibido se correlaciona inversa y significativamente con la desesperanza, aunque en magnitudes bajas a moderadas. Cabe destacar que la dimensión Expectativa de desgracia en el futuro, no se correlaciona significativamente con el apoyo social percibido y sus dimensiones. Igualmente, la dimensión Creencias que los acontecimientos negativos son durables, no se correlaciona con la dimensión Apoyo de familia. Por otro lado, se encontró diferencias significativas del apoyo social percibido con el sexo y la religión, asimismo, la desesperanza con la edad, tiempo de diagnóstico y fase de tratamiento. Conclusión: El apoyo social percibido se relaciona con la desesperanza en pacientes hematooncológicos peruanos. Por tanto, se corrobora que el apoyo social que recibe el paciente ante la desesperanza de una enfermedad hematooncológica, sirve como soporte y tolerancia de dicha enfermedad y sus efectos durante el tratamiento.

Palabras claves: Desesperanza, apoyo social y neoplasias hematológicas.

\section{[en] Hopelessness and its relationship with perceived social support in Peruvian patients with hematological neoplasms}

\footnotetext{
Abstract: Objective: The aim of this study was to determine the significant relationship between social support and hopelessness in Peruvian patients with hematological neoplasms who are in any of the treatment phases. Method: The study was descriptive-correlational. The sample consisted of 110 hemato-oncological patients of both sexes, aged between 16 and 25 years. The instruments used were the Multidimensional Scale of Perceived Social Support (MSPSS) and the Beck Hopelessness Scale

1 Juan Ricardo Peralta Gil. Universidad Peruana Unión, Facultad de Ciencias de la Salud / Escuela Profesional de Psicología. Lima, Perú

E-mail: juanrperalta@upeu.edu.pe

2 Amy Mishelle Mariño Rugel. Universidad Peruana Unión, Facultad de Ciencias de la Salud / Escuela Profesional de Psicología. Lima, Perú

E-mail: amyrugel@upeu.edu.pe

3 Cristian Edwin Adriano Rengifo. Universidad Peruana Unión, Facultad de Ciencias de la Salud / Escuela Profesional de Psicología. Lima, Perú

E-mail: cristianadriano@upeu.edu.pe

* Dirección de correspondencia: Juan Ricardo Peralta Gil. Carretera Central Km 19.5 Naña, Chosica. 15464 Lurigancho. Lima. Perú.

E-mail: juanrperalta@upeu.edu.pe
} 
(BHS). Results: High levels of perceived social support (81\%) and normal levels of hopelessness $(82 \%)$ are reported in hemato-oncological patients. Perceived social support is inversely and significantly correlated with hopelessness, although in low to moderate magnitudes. However, the dimension Expectation of misfortune in the future, does not correlate significantly with the perceived social support and its dimensions. Likewise, the Beliefs that negative events are durable dimension did not correlate with the dimension Family support. On the other hand, significant differences were found in perceived social support with sex and religion, as well as hopelessness with age, time of diagnosis and treatment phase. Conclusion: The perceived social support is related to the hopelessness of Peruvian hemato-oncological patients. Therefore, it is corroborated that the social support that the patient receives when faced with the hopelessness of a hemato-oncological disease, serves as support and tolerance of said disease and its effects during treatment.

Keywords: Hopelessness, social support and hematological neoplasms.

Sumario: 1. Introducción 2. Método 3. Análisis de datos 4. Resultados 5. Discusión 6. Conclusión 7. Referencias bibliográficas

Como citar: Peralta Gil JR, Mariño Rugel AM, Adriano Rengifo CE. Desesperanza y su relación con el apoyo social percibido en pacientes peruanos con neoplasias hematológicas. Psicooncología 2021; 18: 317-332, https://dx.doi.org/10.5209/psic.75631

\section{Introducción}

El cáncer es la segunda causa de muerte en el continente americano, en el 2018, se diagnosticó unos 3,8 millones de casos y a causa de esta enfermedad, fallecieron 1,4 millones de personas ${ }^{(1)}$, por ende, las personas sienten temor ante esta enfermedad crónica que culturalmente se asocia con el dolor, el padecimiento y la muerte ${ }^{(2)}$. Las neoplasias hematológicas en Perú, específicamente, la leucemia durante el período 2010-2012 fue la undécima causa de muerte en Lima metropolitana. Alrededor de 1.076 personas fallecieron a causa de esta enfermedad, con una tasa de mortalidad de 3,9 por cada cien mil habitantes ${ }^{(3)}$. Actualmente, se evidencia un incremento del $50 \%$ en las tasas de incidencia de cánceres tanto en hombres y mujeres ${ }^{(4)}$, y en los niños, las neoplasias hematológicas junto a los tumores del sistema nervioso central son los más frecuentes, en relación a los cánceres hematopoyéticos, el $40,2 \%$ son leucemias. En este sentido, los pacientes con leucemia experimentan afectaciones tanto a nivel físico, social y psicológico ${ }^{(5)}$, que según el grado de amenaza percibido y el apoyo mínimo recibido, supone la presencia de un sufrimiento psicológico significativo $^{(6)}$, este sufrimiento puede perjudicar la calidad de vida, reducir la esperanza de supervivencia y afectar la salud física en general ${ }^{(7,8)}$, es decir, el impacto emocional que se experimenta ante el diagnóstico de cáncer produce en el individuo una reconceptualización de la esperanza, la cual puede variar en intensidad según sea el impacto. En otras palabras, existe la posibilidad de que la esperanza se torne en desesperanza por el fuerte impacto percibido y el estado de vulnerabilidad psicológico actual ${ }^{(9)}$. La desesperanza, entre otras variables intrapersonales, es la reacción psicológica más común en pacientes oncológicos ${ }^{(10)}$, por lo que impacta directamente en el bienestar físico y mental de las personas ${ }^{(11)}$, además de estar relacionada con el deseo de una muerte acelerada ${ }^{(12)}$. Según Beck ${ }^{(13)}$, la desesperanza es la actitud o expectativa negativa acerca del futuro donde predomina los sentimientos de desesperación, decepción y desilusión, también, la considera como un patrón cognitivo consistente sobre las atribuciones negativas hacia el futuro. 
Por otro lado, el apoyo social percibido por el paciente, reduce la tensión, aumenta la tasa de supervivencia y mejora el estado de salud y calidad de vida ${ }^{(14)}$, por ello, los pacientes que asisten a pruebas de diagnóstico y procedimientos en compañía de terceros, perciben mayor soporte emocional durante la terapia contra el cáncer ${ }^{(15)}$. Se ha definido que el apoyo social es una apreciación personal sobre el apoyo otorgado por la red social ${ }^{(16)}$, Garcés y Durá ${ }^{(17)}$ lo corroboran, ya que este recurso psicológico es uno de los métodos efectivos para brindar soporte a los pacientes, tanto a nivel cognitivo; que consiste en presentar información sobre la afección y el tratamiento, a nivel emocional; que busca afrontar las reacciones emocionales generadas por la enfermedad, y conductual; que intenta brindar estrategias concretas para confrontar los problemas; inclusive, se ha demostrado que altos niveles de soporte social conlleva a la mejora de la salud y disminución de la mortalidad, mientras que su ausencia se refleja en la prevalencia del cáncer y una disminución de la supervivencia ${ }^{(18)}$, motivo por el cual, los pacientes oncológicos valoran las distintas fuentes de apoyo social percibido, con el fin de reducir el estrés ocasionado por la incertidumbre hacia el futuro ${ }^{(19)}$.

Incrustados a esta realidad y según estudios a nivel internacional se evidencia que la desesperanza asociado con la enfermedad puede ser influenciada por el apoyo social percibido ${ }^{(20)}$. Según un estudio de relación entre apoyo social y desesperanza en pacientes oncológicos de un hospital de Turquía, se encontró que existe una correlación negativa entre apoyo social y desesperanza, y que la familia cumple un rol fundamental en la vida del paciente ${ }^{(21)}$. En Irán, tras encontrar que existe correlaciones negativamente significativas entre las puntuaciones de desesperanza y apoyo social por parte de sus familiares y amigos, sus hallazgos muestran que, los pacientes iraníes evidencian niveles leves de desesperanza y niveles moderados de apoyo social, y que, el apoyo social percibido influye en la esperanza de los pacientes $^{(22)}$. Mientras que, otros estudios ${ }^{(20,23)}$ han analizado su relación en conjunto con otros factores, o bien, una comparación con los niveles de desesperanza de sus cuidadores, evidenciándose el bajo aporte de estudios sobre la relación de estos dos constructos en pacientes con neoplasias hematológicas.

Por tanto, considerando que existen estudios sobre la relación entre ambas variables y que existe un bajo aporte de investigaciones que describan la difícil situación de estos pacientes en los países en desarrollo, como Perú. El presente estudio tiene como objetivo principal analizar la relación entre el apoyo social percibido y la desesperanza en pacientes peruanos con neoplasias hematológicas.

\section{Método}

\section{Diseño y tipo de investigación}

Esta investigación es de diseño no experimental y de corte transversal, porque no se manipularon las variables y los datos fueron tomados en un momento determinado. Asimismo, es de tipo descriptivo correlacional ${ }^{(24)}$.

\section{Participantes}

Se contó con la participación de 110 personas diagnosticadas con cáncer en la sangre, de ambos sexos, de edades de 16 a 25 años, con tiempo de diagnóstico mínimo de 
3 meses y que se encuentren en tratamiento. El muestreo fue no probabilístico de tipo bola de nieve, en la que se identificaron a participantes claves que se incluyeron en la muestra, luego se les preguntó si conocen a otras personas con características similares para ser contactados e incluidos a la muestra ${ }^{(24)}$. En la tabla 1 se proporciona la información sociodemográfica de los participantes.

\section{Procedimiento}

En primer lugar, se identificó a los posibles candidatos. Luego de verificar que los candidatos cumplían con los criterios de inclusión de la muestra se procedió a contactarlos por teléfono o redes sociales (Facebook, WhatsApp, Email), debido al contexto sanitario que se vive en el país ocasionado por la COVID-19. Luego de explicar los objetivos y todos los lineamientos éticos de la investigación a los candidatos, indicaron su consentimiento para participar en el estudio aceptando completar los formularios virtuales (Google Forms). Para evitar resultados sesgados por los efectos del tratamiento, solo se pidió a los candidatos que participaran en el estudio si su estado de salud general era adecuado. La aplicación se llevó a cabo del 2 de junio al 4 de julio del 2020.

\section{Instrumentos}

Ficha de Datos Sociodemográficos

Se empleó una Ficha de Registro elaborada por los mismos autores en la que se recogieron datos sobre las características sociodemográficas como el sexo, edad, religión que profesa. Además, datos relacionados con la enfermedad como tipo de diagnóstico de alguna enfermedad hematooncológica, tiempo desde que recibió el diagnóstico, fase de tratamiento en la que se encuentra y si tuvo recaídas. Por último, que indicara la(s) persona(s) que le brinda el mayor apoyo desde que recibió el diagnóstico.

\section{Escala Multidimensional de Apoyo Social Percibido}

Escala Multidimensional de Apoyo Social Percibido (MSPSS, por sus siglas en inglés) fue creada por Zimet et al. ${ }^{(25)}$ y adaptada al español por Landete y Calvete ${ }^{(26)}$. Se utilizó la escala adaptada a Perú por Juárez ${ }^{(27)}$ para evaluar el apoyo social percibido de las personas. Se administra en adolescentes, jóvenes y adultos. La escala tiene un total de 12 ítems, conformada por tres subescalas (Familia, Amigos y Otros significativos) cada una compuesta por 4 ítems, con 6 opciones de respuestas de tipo Likert (Totalmente de acuerdo, Bastante de acuerdo, De acuerdo, En desacuerdo, Bastante en desacuerdo y Totalmente en desacuerdo), que se mide en una escala de 1 a 6 puntos. Puntajes mayores indican mayor apoyo social percibido. Además, la escala presenta tres niveles de categorías de apoyo social percibido; bajo, medio y alto. Según el estudio de Juarez ${ }^{(27)}$, la escala evidencia validez en su estructura interna de un modelo no jerárquico oblicuo con 12 ítems y 3 factores, con adecuados ajustes al modelo $(\mathrm{X} 2 / \mathrm{df}=1,6, \mathrm{P}=0, \mathrm{GFI}=, 94, \mathrm{CFI}=, 98, \mathrm{SRMR}=, 03$, RMSEA $=, 05$, AIC $=137,084$ ). Asimismo, presenta adecuados coeficientes de confiabilidad mediante el omega de McDonald (familia $\omega=, 88$, amigos $\omega=, 94$, otros 
significativos $\omega=, 86)$. Los índices de confiabilidad obtenidos en la muestra de este estudio fueron para familia $\alpha=, 94$, amigos $\alpha=, 97$, otros significativos $\alpha=, 96$ ).

\section{Escala de Desesperanza de Beck}

Escala de Desesperanza de Beck (BHS, por sus siglas en inglés) fue creada por Beck et al. ${ }^{(28)}$ para medir el grado de patrones cognitivos característicos, concerniente en atribuciones negativas acerca del futuro del sujeto. La escala se puede administrar a jóvenes y adultos y tiene una duración aproximadamente entre 5 a 10 minutos. En esta investigación se utilizó la adaptación a Lima-Perú de Aliaga et al. ${ }^{(29)}$. Ésta versión está compuesta por 18 ítems con un tipo de respuesta dicotómico de verdadero o falso, que se miden en 0 y 1 . La calificación de la escala oscila entre 0 a 18, otorgándole un punto al ítem que señala desesperanza y cero a quienes no. Según la interpretación de Beck \& Steer, de 0 a 2 equivale a un rango normal de desesperanza, de 3 a 4 leve de desesperanza, de 5 a 7 moderada desesperanza y de 8 a 18 severa desesperanza. Para la validez de la escala utilizaron la validez factorial obteniendo 6 factores que explican el 49,32\% de la varianza acumulada, asimismo, en la validez concurrente encontraron correlaciones positivas adecuadas con la Escala de Depresión de Beck (BDI); esto evidencia adecuada validez del constructo. En cuanto a la confiabilidad, la estimaron mediante el coeficiente alfa de Cronbach $(, 76)$ y mediante el coeficiente de correlación de Pearson de los puntajes del test-retest $(, 68)$ con un intervalo de dos semanas. Para la muestra de pacientes hematooncológicos de este estudio se reportó un índice de confiabilidad de $\alpha=, 88$ en la escala global.

\section{Análisis estadísticos}

Los datos recolectados se analizaron utilizando el programa estadístico IBM SPSS24. Para clasificar y resumir los hallazgos se utilizaron estadísticos descriptivos, incluyendo tablas de frecuencia absoluta y relativa, medias, desviación estándar e intervalos de confianza para reportar el perfil sociodemográfico. Se utilizó la prueba de normalidad Kolmogorov-Smirnov para determinar la distribución de los datos. Para los análisis inferenciales se utilizó el coeficiente de correlación de Spearman para determinar las correlaciones entre las variables de estudio, $U$ de Mann-Whitney y Kruskal-Wallis para determinar las diferencias entre grupos.

\section{Resultados}

En la tabla 1 se aprecia que de los 110 pacientes con neoplasias hematológicas que participaron en el estudio, 72 (66\%) son varones. El 61\% se encuentra entre 18 a 23 años de edad. El 54\% profesa la fe católica. El 78\% presentó un diagnóstico de Leucemia. También el $47 \%$ pressenta más de dos años de enfermedad. El $60 \%$ se encontraba en la fase de mantenimiento del tratamiento. El 58\% refirió no haber tenido recaídas en la enfermedad. El $82 \%$ señaló como fuente de mayor apoyo a sus padres.

Por otro lado, en la tabla 1 se evidencia que el sexo y la religión fueron variables sociodemográficas que presentan diferencia significativa con el apoyo social. Los pacientes de sexo femenino recibieron mayor nivel de apoyo social que los pacientes 
varones. Además, se encontró que los pacientes que profesan la fe evangélica presentaron mayores niveles de apoyo social.

También se puede apreciar que la edad, el tiempo de diagnóstico y la fase del tratamiento son variables que presentan diferencias significativas con la desesperanza. Los pacientes más jóvenes (16 a 17 años) presentan mayores niveles de desesperanza que los pacientes de más edad. Asimismo, los pacientes que fueron recientemente diagnosticados ( $<6$ meses) presentan mayores niveles de desesperanza. Además, los pacientes que se encuentran en la fase de inducción del tratamiento presentan mayores niveles desesperanza que aquellos pacientes que se encuentran en las fases de consolidación y mantenimiento.

Tabla 1. Perfil sociodemográfico y sus efectos sobre el Apoyo social y Desesperanza en pacientes hematooncológicos

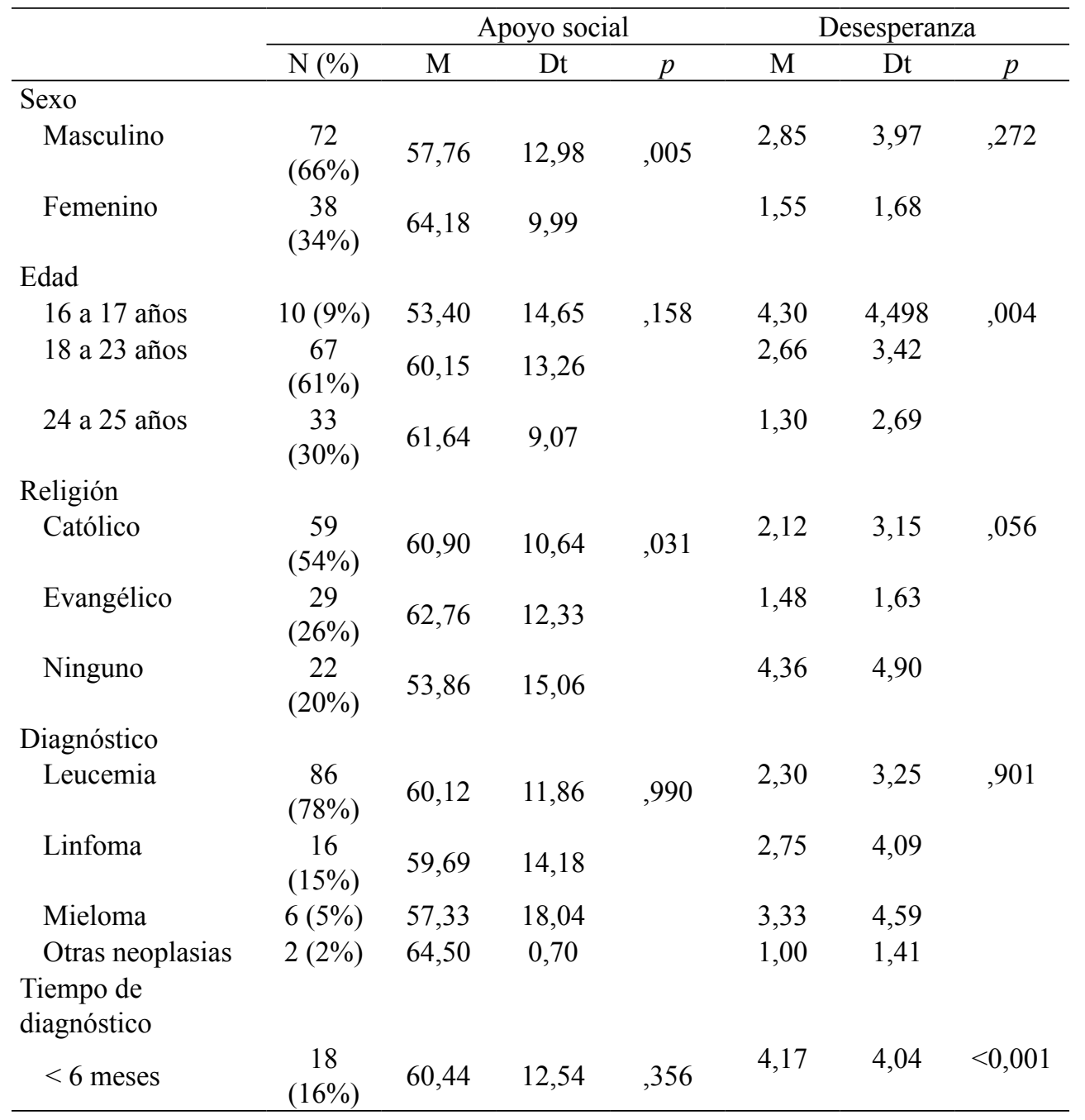




\begin{tabular}{|c|c|c|c|c|c|c|c|}
\hline & \multicolumn{4}{|c|}{ Apoyo social } & \multicolumn{3}{|c|}{ Desesperanza } \\
\hline & $\mathrm{N}(\%)$ & M & Dt & $p$ & $\mathrm{M}$ & Dt & $p$ \\
\hline 6 meses a 1 año & $\begin{array}{c}18 \\
(16 \%)\end{array}$ & 61,67 & 12,12 & & 2,39 & 2.50 & \\
\hline 1 a 2 años & $\begin{array}{c}23 \\
(21 \%)\end{array}$ & 56,87 & 12,54 & & 2,87 & 3,40 & \\
\hline 2 años a más & $\begin{array}{c}51 \\
(47 \%)\end{array}$ & 60,63 & 12,45 & & 1,57 & 3,27 & \\
\hline \multicolumn{8}{|l|}{ Fase tratamiento } \\
\hline Inducción & $\begin{array}{c}19 \\
(17 \%)\end{array}$ & 61,95 & 13,22 & ,406 & 3,89 & 4,08 & ,006 \\
\hline Consolidación & $\begin{array}{c}25 \\
(23 \%)\end{array}$ & 59,64 & 10,41 & & 2,48 & 3,33 & \\
\hline Mantenimiento & $\begin{array}{c}66 \\
(60 \%)\end{array}$ & 59,55 & 12,92 & & 1,94 & 3,15 & \\
\hline \multicolumn{8}{|l|}{ Recaídas } \\
\hline Sí & $\begin{array}{c}46 \\
(42 \%)\end{array}$ & 58,07 & 12,55 & ,102 & 2,96 & 4,04 & ,319 \\
\hline No & $\begin{array}{c}64 \\
(58 \%)\end{array}$ & 61,36 & 12,15 & & 2,00 & 2,84 & \\
\hline \multicolumn{8}{|l|}{$\begin{array}{l}\text { Fuente de mayor } \\
\text { apoyo }\end{array}$} \\
\hline Padres & $\begin{array}{c}90 \\
(82 \%)\end{array}$ & 60,47 & 12,07 &, 170 & 2,40 & 3,35 & ,154 \\
\hline Pareja & $\begin{array}{c}11 \\
(10 \%)\end{array}$ & 63,27 & 8,38 & & 1,00 & 1,26 & \\
\hline Cuidador & $7(6 \%)$ & 54,86 & 16,99 & & 3,86 & 5,69 & \\
\hline Otros & $2(2 \%)$ & 38,00 & 1,41 & & 5,00 & 2,82 & \\
\hline
\end{tabular}

En la tabla 2 se visualiza que la mayoría de los pacientes hematooncológicos reportan niveles altos de apoyo de la familia, amigos y otras personas significativas. En general, el apoyo social resultó encontrarse en niveles elevados. En cuanto a la desesperanza y sus dimensiones se encontró niveles normales y leves en los pacientes hematooncológicos.

Tabla 2. Estadísticos descriptivos de las variables de estudio

\begin{tabular}{lcccc}
\hline & N $(\%)$ & M & Dt & IC 95\% \\
\hline Apoyo de familia & & & & \\
Bajo & $12(11 \%)$ & & & \\
Medio & $7(6 \%)$ & 20,72 & 4,62 & $19,84-21,54$ \\
Alto & $91(83 \%)$ & & & \\
\hline
\end{tabular}




\begin{tabular}{|c|c|c|c|c|}
\hline & N (\%) & M & Dt & IC $95 \%$ \\
\hline \multicolumn{5}{|c|}{ Apoyo de amigos } \\
\hline Bajo & $8(7 \%)$ & & & \\
\hline Medio & $24(22 \%)$ & 19,65 & 4,91 & $18,78-20,60$ \\
\hline Alto & $78(71 \%)$ & & & \\
\hline \multicolumn{5}{|c|}{ Apoyo de otros } \\
\hline Bajo & $19(17 \%)$ & & & \\
\hline Medio & $17(16 \%)$ & 19,61 & 5,56 & $18,54-20,64$ \\
\hline Alto & $74(67 \%)$ & & & \\
\hline \multicolumn{5}{|c|}{ Apoyo social global } \\
\hline Bajo & $10(9 \%)$ & & & \\
\hline Medio & $11(10 \%)$ & 59,98 & 12,37 & $57,68-62,38$ \\
\hline Alto & $89(81 \%)$ & & & \\
\hline \multicolumn{5}{|c|}{$\begin{array}{l}\text { Carencia de ilusión en el } \\
\text { futuro }\end{array}$} \\
\hline Normal & $103(93 \%)$ & & & \\
\hline Leve & $4(4 \%)$ & & & \\
\hline Moderado & $1(1 \%)$ &, 55 & 1,28 &, $35-, 82$ \\
\hline Grave & $2(2 \%)$ & & & \\
\hline \multicolumn{5}{|c|}{$\begin{array}{l}\text { Expectativa de infelicidad } \\
\text { en el futuro }\end{array}$} \\
\hline Normal & $68(62 \%)$ & & & \\
\hline Leve & $23(21 \%)$ & & & \\
\hline Moderado & $9(8 \%)$ & ,70 & 1,12 &, $50-, 92$ \\
\hline Grave & $10(9 \%)$ & & & \\
\hline \multicolumn{5}{|c|}{$\begin{array}{l}\text { Expectativa de desgracia } \\
\text { en el futuro }\end{array}$} \\
\hline Normal & $104(94 \%)$ & & & \\
\hline Leve & $5(5 \%)$ & & & \\
\hline Moderado & $1(1 \%)$ &, 06 & ,28 &, $02-, 12$ \\
\hline Grave & $0(0 \%)$ & & & \\
\hline \multicolumn{5}{|c|}{$\begin{array}{l}\text { Expectativa de un futuro } \\
\text { incierto }\end{array}$} \\
\hline Normal & $59(54 \%)$ & & & \\
\hline Leve & $21(19 \%)$ & & & \\
\hline Moderado & $20(18 \%)$ &, 83 & 1,03 & $, 65-1,04$ \\
\hline Grave & $10(9 \%)$ & & & \\
\hline \multicolumn{5}{|c|}{$\begin{array}{l}\text { Creencia que los aconte- } \\
\text { cimientos negativos son } \\
\text { durables }\end{array}$} \\
\hline Normal & $97(88 \%)$ & & & \\
\hline Leve & $13(12 \%)$ & & & \\
\hline Moderado & $0(0 \%)$ & ,12 &, 32 &, $06-, 18$ \\
\hline Grave & $0(0 \%)$ & & & \\
\hline
\end{tabular}




\begin{tabular}{lcccc}
\hline & N $(\%)$ & M & Dt & IC 95\% \\
\hline $\begin{array}{l}\text { Creencia en la imposibili- } \\
\text { dad de ser favorecido por } \\
\text { el azar }\end{array}$ & & & \\
Normal & $95(86 \%)$ & & \\
Leve & $15(14 \%)$ & &, 34 &, $07-, 21$ \\
Moderado & $0(0 \%)$ &, 14 & & \\
Grave & $0(0 \%)$ & & & \\
Desesperanza Global & & & \\
Normal & $90(82 \%)$ & & \\
Leve & $12(11 \%)$ & & \\
Moderado & $5(4 \%)$ & 2,40 & 3,41 & \\
Grave & $3(3 \%)$ & & & \\
\hline
\end{tabular}

En la tabla 3 se aprecia que el Apoyo social y sus dimensiones se correlacionan inversa y significativamente con la desesperanza y sus dimensiones. Se puede notar que estas correlaciones presentan magnitudes bajas y moderadas, por lo que su interpretación debe considerarse con precaución.

No obstante, se observa que la dimensión Expectativa de desgracia en el futuro no se correlaciona con Apoyo social $(r=-0,161 p>0,05)$, Apoyo familiar $(r=-0,159$ $p>0,05)$, Apoyo de amigo $(r=-0,163 p>0,05)$ y Apoyo de otros $(r=-0,168 p$ $>0,05)$. Igualmente, la dimensión Creencia que los acontecimientos negativos son durables no se correlaciona con Apoyo familiar $(r=-0,081 p>0,05)$.

Finalmente, el Apoyo social global se correlaciona inversamente y muestra significancia estadística con la desesperanza $(r=-0,522 p<0,01)$. Esto indica que, a mayor presencia de apoyo social, menor será la desesperanza en los pacientes hematooncológicos.

Tabla 3. Correlaciones entre las variables de estudio

\begin{tabular}{lcccc}
\hline & $\begin{array}{c}\text { Apoyo } \\
\text { Social }\end{array}$ & $\begin{array}{c}\text { Apoyo de } \\
\text { familia }\end{array}$ & $\begin{array}{c}\text { Apoyo de } \\
\text { amigos }\end{array}$ & $\begin{array}{c}\text { Apoyo de } \\
\text { otros }\end{array}$ \\
\hline Desesperanza &,$- 522^{* *}$ &,$- 368^{* *}$ &,$- 502 * *$ &,$- 437 * *$ \\
Carencia de ilusión en el futuro &,$- 421^{* *}$ &,$- 448^{* *}$ &,$- 344^{* *}$ &,$- 342 * *$ \\
Expectativa de infelicidad en el futuro &,$- 405^{* *}$ &,$- 332 * *$ &,$- 388^{* *}$ &,$- 349 * *$ \\
Expectativa de desgracia en el futuro &,- 161 &,- 159 &,- 163 &,- 168 \\
Expectativa de un futuro incierto &,$- 485^{* *}$ &,$- 345^{* *}$ &,$- 448^{* *}$ &,$- 425^{* *}$ \\
$\begin{array}{l}\text { Creencia que los acontecimientos } \\
\text { negativos son durables }\end{array}$ &,$- 260^{* *}$ &,- 081 &,$- 244 *$ &,$- 281^{* *}$ \\
$\begin{array}{l}\text { Creencia en la imposibilidad de ser } \\
\text { favorecido por el azar }\end{array}$ &,$- 257 * *$ &,$- 316^{* *}$ &,$- 207 *$ &,$- 207 *$ \\
\hline
\end{tabular}

$\mathrm{N}=110$.

$*$ La relación es significativa $<, 05$

$* *$ La relación es significativa $<, 01$ 


\section{Discusión}

El presente estudio tuvo como objetivo determinar la relación entre la desesperanza y el apoyo social percibido en una muestra de pacientes peruanos con neoplasias hematológicas que se encuentren en alguna de las fases del tratamiento. De acuerdo con los resultados encontrados, la mayoría de los pacientes presentaron niveles moderados $(10 \%)$ y altos $(81 \%)$ de apoyo social. Estos resultados se asemejan con el estudio de Pérez et al. ${ }^{(30)}$, quienes reportaron que el $76.8 \%$ de los pacientes oncológicos colombianos alcanzaron un puntaje medio en apoyo emocional/informacional y un $23.2 \%$ obtuvo un puntaje alto. De la misma manera, pacientes oncológicos iraníes mostraron niveles moderados de apoyo social ${ }^{(22)}$. Por otro lado, se encontró que la mayoría de los pacientes reportaron niveles en rangos normales (82\%) y leves (11\%) de desesperanza. Esto se asemeja con diferentes estudios ${ }^{(31-33)}$ en que reportaron niveles normales y/o leve de desesperanza en los pacientes oncológicos. Esto sugiere que los pacientes oncológicos suelen percibir adecuadamente el apoyo social de su entorno cercano, lo que favorece la disminución de la desesperanza frente al diagnóstico de esta neoplasia.

Conforme a lo esperado, se encontró que la desesperanza tiene relación inversa altamente significativa con el apoyo social y cada una de sus dimensiones; es decir, los pacientes con neoplasias hematológicas que perciben un alto apoyo social por parte de su entorno, presentan bajos niveles de desesperanza. En otras palabras, no presentan o manifiestan en menor intensidad actitudes y expectativas negativas acerca de su futuro. Por consiguiente, el apoyo brindado por parte de familiares, amigos y miembros terapéuticos juega un papel importante en la vida del paciente, ya que favorece a la aceptación de la enfermedad y adherencia al tratamiento ${ }^{(22,34)}$, además de que actúa como un amortiguador hacia el estrés de la enfermedad y a la vez, ejerce un efecto inmunoprotector ${ }^{(35,36)}$.

Al analizar cada una de las dimensiones de apoyo social, se encontró relación inversa altamente significativa $\left(\mathrm{rho}=-, 368^{* *}, \mathrm{p}<0,01\right)$ entre el apoyo de la familia y la desesperanza; es decir, a mayor apoyo familiar, menor son las actitudes o expectativas negativas acerca del futuro. Esto concuerda con la teoría de la desesperanza de la depresión que plantea que, ante la presencia de eventos negativos y la falta de apoyo social conlleva a niveles altos de desesperanza, por lo tanto, desencadena el síndrome de depresión desesperada ${ }^{(37)}$. En este contexto, el esfuerzo y el apoyo de los miembros de la familia puede brindar esperanza y sentimientos de confianza a los pacientes, y una razón para aferrarse a la vida, debido al aumento de energía para luchar contra la enfermedad. De manera que, el apoyo de los familiares, fortalece la creencia y el sentido de vida del paciente, facilita la adaptación al cáncer tanto física como psicológica, ya que, en un período de crisis, la mayoría requiere de familiares de confianza ${ }^{(34)}$, inclusive esto se ve reflejado en las propias familias con hijos con cáncer, que experimentan bajos niveles de desesperanza, cuando perciben niveles altos de apoyo social ${ }^{(38)}$. Específicamente, en pacientes con leucemia aguda se destaca la importancia del apoyo social durante el tratamiento, debido a la necesidad de soporte del día a día y al espacio de reflexión que encuentran en ellos al meditar sobre su nueva situación de vida ${ }^{(39)}$.

En el presente estudio, se consideró a la familia como la mayor fuente de apoyo social para los pacientes con neoplasias hematológicas. Otro estudio, así lo corrobora, como la principal fuente de apoyo para los pacientes con cáncer ${ }^{(40)}$. Esto 
se debe a que, generalmente, la mayoría de los pacientes necesitan de su familia por varios aspectos funcionales, como; necesidades fisiológicas y de seguridad. Particularmente, los padres son entes principales de apoyo social, que abarca; al apoyo emocional, apoyo instrumental e informativo ${ }^{(41)}$.

Respecto a la dimensión apoyo de amigos, se encontró que existe relación inversa altamente significativa ( $\left.r h o=-, 502^{* *}, \mathrm{p}<0,01\right)$ con la desesperanza, lo que significa que, a mayor apoyo social de los amigos, menor son las actitudes o expectativas negativas hacia el futuro. A medida que los adolescentes maduran y se convierten en adultos jóvenes, gradualmente se tornan más independientes de su familia. Si bien muchos adolescentes y adultos jóvenes continúan dependiendo de los padres, especialmente en relación con el apoyo instrumental, suelen también expandir sus redes sociales durante su hospitalización y/o tratamiento, y desarrollar relaciones de mayor apoyo social con sus compañeros ${ }^{(40)}$. Interactuar con amigos permite a los supervivientes reintegrarse de nuevo en la vida normal. La capacidad de mantener amistades durante el tratamiento permite, específicamente a los adolescentes, superar los desafíos y los efectos residuales del tratamiento oncológico. Galán et al. ${ }^{(42)}$ mencionan que los problemas psicosociales que enfrentan los supervivientes, como el miedo de perder el contacto y la incapacidad de socializar con amigos, pueden conducir al aislamiento y actuar como una barrera para volver a entrar en la normalidad. Además, descubrieron que es importante contar con el apoyo de los amigos, especialmente aquellos con experiencias similares al cáncer, ya que, el compartir vivencias sobre los síntomas del tratamiento, el proceso de recuperación y la manera en cómo se maneja la situación como pacientes, proporciona esperanza para el futuro y la convicción de poder hacer frente al tratamiento ${ }^{(39)}$.

En cuanto a la dimensión apoyo de 'otras personas significativas', se encontró que existe relación inversa altamente significativa $\left(r h o=-, 437^{* *}, p<0,01\right)$ con la desesperanza, es decir que, a mayor soporte de otras personas significativas de su entorno, como cuidadores, profesionales de la salud y vecinos, menor son las actitudes o expectativas hacia el futuro. Los cuidadores de un estudio describieron "al hogar como una fuente de sanación y esperanza, donde los pacientes pueden mejorar si no es físicamente, al menos mentalmente" ${ }^{\prime(43, p .23)}$. En efecto, uno de los factores importantes en el pronóstico del tratamiento, es la ausencia de la desesperanza, esta permite vivir con tranquilidad, brinda seguridad y además ayuda al paciente a deshacerse de sentimientos como el pesimismo y el desamparo, componentes que influyen en adaptación al tratamiento. Es así que el apoyo social recibido de personas significativas aumenta la respuesta de espíritu de lucha entre los pacientes con cáncer ${ }^{(34)}$. Por lo que, el abordaje de tratamientos psicológicos como; las técnicas de relajación e imaginación, la terapia expresiva de apoyo y los grupos de apoyo, son técnicas terapéuticas que disminuyen los efectos secundarios causados por la quimioterapia y radioterapia ${ }^{(4)}$. Además, las intervenciones en término de bienestar psicológico, donde se fortalece el "deseo de vivir", el enfrentamiento de la desesperanza y la presencia de grupos de apoyo, entre otros, producen una mejoría incuestionable en la calidad de vida del paciente ${ }^{(44)}$.

De manera general, se ha demostrado que el apoyo social, disminuye la sensación de desesperanza y soledad, y que además tiene un efecto positivo en el bienestar psicológico y sentido de vida del paciente ${ }^{(45,46)}$. Asimismo, se considera que el apoyo social tanto de familiares, amigos y otros pacientes es invaluable y actúa como salvavidas en el tratamiento, dado que disminuye los niveles de desesperanza ${ }^{(47)}$. 
Con el propósito de profundizar en los análisis, en este estudio se examinaron las diferencias del apoyo social percibido según el perfil sociodemográficos de los pacientes con neoplasias hematológicas. Se encontró que solo la variable sexo y religión presentan diferencias significativas con el apoyo social percibido. Fueron las mujeres que recibieron mayor apoyo social a diferencia de los varones. Este hallazgo es consistente con el estudio de Yuo y $\mathrm{Lu}^{(48)}$, en el que señalan que las mujeres tienden a percibir mayor apoyo social que los hombres. Igualmente, el estudio de Pérez et al. ${ }^{(30)}$ sostiene que son las mujeres las que perciben mayor apoyo social, aunque los autores señalan que tuvieron una mayor muestra de mujeres que de hombres, lo que pudo haber afecto su resultado. Cabe resaltar que este estudio contó con una muestra menor de mujeres que de hombres. Este hallazgo puede sugerir que realmente son las mujeres que perciben mayor apoyo social de su entorno. También se encontró que los pacientes que profesan la fe evangélica mostraron mayores niveles de apoyo social que los católicos y aquellos que no tienen ninguna filiación religiosa. Esto coincide con diversos estudios ${ }^{(49-51)}$ que plantean que las creencias espirituales y el sentido de pertenencia a una comunidad religiosa en pacientes oncológicos, es una fuente que facilita el apoyo social, y por ende mejora la salud mental.

Por otro lado, la desesperanza mostró diferencias estadísticamente significativas con la edad, tiempo de diagnóstico y fase de tratamiento en la se encuentra el paciente con neoplasias hematológicas. Los pacientes más jóvenes (16 a 17 años) mostraron mayores niveles de desesperanza a diferencia de los pacientes con más edad. Esto se corrobora con algunos investigadores, como Benzein y Berg( ${ }^{(52)}$ y Okano et al. ${ }^{(53)}$ que encontraron relación negativa estadísticamente significativa entre la edad y los niveles de desesperanza, es decir, a medida que los pacientes iban aumentando de edad, el nivel de desesperanza disminuía.

Los pacientes que tenían poco tiempo de diagnóstico $(<6$ meses $)$ presentaron mayores niveles de desesperanza que aquellos con más tiempo de diagnóstico, lo que difiere con el estudio de Garcés ${ }^{(31)}$, quien encontró que los pacientes con un tiempo de diagnóstico menor a seis meses, presentaban niveles bajos de desesperanza, incluso, pacientes ambulatorios con cáncer metastásico reportaron hasta un $23 \%$ de niveles moderados a severos de desesperanza ${ }^{(54)}$. Los pacientes que se encontraban en la fase de inducción del tratamiento mostraron mayores niveles de desesperanza a comparación de aquellos que se encontraban en la fase de consolidación o mantenimiento. Esto se contradice con el estudio de Greihman et al. ${ }^{(55)}$, que entre sus resultados se evidencia sorprendentemente que la desesperanza es menos común en la etapa inicial de la leucemia aguda.

\section{Conclusión}

De acuerdo a los resultados de esta investigación, se puede concluir que los pacientes con neoplasias hematológicas reportaron niveles altos de apoyo social y rangos normales de desesperanza. También, se encontró que las mujeres presentan mayor nivel de apoyo social que los varones. Además, los pacientes que profesan la fe evangélica mostraron mayores niveles de apoyo a diferencia de los católicos y aquellos que no tienen ninguna filiación religiosa. Por otro lado, los pacientes más jóvenes (16 a 17 años), recientemente diagnosticados ( $<6$ meses) y en la fase de inducción del tratamiento mostraron mayores niveles de desesperanza. Por último, 
se comprobó que el apoyo social percibido tiene relación inversa significativa con la desesperanza en pacientes con neoplasias hematológicas.

Esta investigación representa un aporte al campo de la psicooncología sobre el papel fundamental que cumple el apoyo social en los pacientes oncológicos, especialmente a los de neoplasias hematológicas, lo que favorece la disminución de la desesperanza frente al diagnóstico de esta neoplasia. Es necesario seguir realizando más investigaciones en los pacientes oncológicos peruanos para fortalecer los resultados hallados en este estudio, empleando una muestra mayor y con tipo de muestreo probabilístico. También realizar estudios de tipo predictivo para analizar el grado de influencias de las variables de estudio.

Algunas limitaciones de este estudio fue no contar con una muestra grande de participantes, debido a un diagnóstico oncológico específico. Además, los resultados hallados no se pueden generalizar, debido al tipo de muestreo no probabilístico.

\section{Referencias bibliográficas}

1. Organización Panamericana de la Salud - OPS. Programa de Cáncer. Organización Panamericana de la Salud. En ILínea [Acceso el 15 de abril de 2020]. Disponible en: https://www.paho.org/es/temas/cancer

2. Die Trill M. Psicooncología. Madrid: ADES, 2003.

3. Instituto Nacional de Enfermedades Neoplásicas - INEN. Registro de cáncer de Lima Metropolitana: Volumen V, Estudio de incidencia y mortalidad 2010-2012. En Línea [Acceso el 10 de abril de 2020]. Disponible en: http://www.inen.sld.pe/portal/ documentos/pdf/banners_2014/2016/Registro\%20de\%20C\%C3\%A1ncer\%20Lima $\% 20$ Metropolitana\%202010\%20-\%202012_02092016.pdf

4. Huerta E. Más de 70 mil casos de cáncer se reportarán en el Perú durante el 2020. RPP. En línea [Acceso el 4 de abril de 2020]. Disponible en: https://rpp.pe/vital/salud/diamundial-contra-el-cancer-mas-de-70-mil-casos-de-neoplasias-se-reportaran-en-el-perudurante-el-2020-noticia-1243382

5. Tomaszewski EL, Fickley CE, Maddux L, Krupnick R, Bahceci E, Paty J, van Nooten F. The patient perspective on living with acute myeloid leukemia. Oncol Ther 2016; 4:225238. https://doi.org/10.1007/s40487-016-0029-8

6. Troncoso P, Rydall A, Rodin G. Psicooncología en cáncer avanzado. Terapia CALM, una intervención canadiense. Neuro Psiquiat 2019; 57:238-46. https://doi.org/10.4067/ S0717-92272019000300238

7. McClement SE, Chochinov HM. Hope in advanced cancer patients. Eur J Cancer 2008; 44:1169-74. https://doi.org/10.1016/j.ejca.2008.02.031

8. Spangenberg L, Zenger M, García-Torres F, Mueller V, Reck M, Mehnert A, Vehling S. Dimensionality, Stability, and Validity of the Beck Hopelessness Scale in Cancer Patients Receiving Curative and Palliative Treatment. J Pain Symptom Manage 2016; 51:615-22. https://doi.org/10.1016/j.jpainsymman.2015.11.008

9. Ripamonti CI, Miccinesi G, Pessi MA, Di Pede P, Ferrari M. Is it posible to encourage hope in non-advanced cancer patients? We must try. Ann Oncol 2015; 27:513-519. https:// doi.org/10.1093/annonc/mdv614

10. Dansuk R, Agargün M, Kars B, Agargün H, Turan C, Ünal O. Evaluation of the psychosocial characteristics of gynaecologic cancer patients. Clin Obstet Gynaecol 2002; 12:142-7. 
11. Grassi L, Travado L, Gil F, Sabato S, Rossi E, Tomamichel M, Marmai L, Biancosino B, Nanni MG. Hopelessness and related variables among cancer patients in the Southern European Psycho-Oncology study (SEPOS). Psychosomatics 2010; 51: 201-7. https:// doi.org/10.1176/appi.psy.51.3.201

12. Mehnert A, Brähler E, Faller H, Härter M, Keller M, Schulz H, Wegscheider K et al. Four-Week prevalence of mental disorders in patients with cancer across major tumor entities. J Clin Oncol 2014; 32:3540-6. https://doi.org/10.1200/JCO.2014.56.0086

13. Beck A. Hopelessness and Suicidal Behavior. JAMA. 1975; 234:1146-1149.

14. Heiydari S, Salahshorian A, Rafie F, Hoseini F. Correlation of perceived social support and size of social network with quality of life dimension in cancer patients. FEYZ 2008; $12: 15-22$.

15. Williams G, Pisu M, Rocque G, Williams C, Taylor R, Kvale E, Partridge E, Bhatia S, Kenzik K. Unmet Social Support Needs Among Older Adults With Cancer. The Oncologist 2019; 125: 473-81. https://doi.org/10.1002/cncr.31809

16. Barra E. Apoyo social, estrés y salud. Psicología y Salud 2004; 14:237-43. Https://doi. org/10.25009/pys.v14i2.848

17. Garcés J, Durá E. La teoría del apoyo social y sus implicaciones para el ajuste psicosocial de los enfermos oncológicos. Rev Psicol Social 1991; 6: 257-71.

18. Kadambi S, Soto-Perez-de-Celis E, Garg T, Loh K, Krok-Schoen J, Battisti N, et al. Social support for older adults with cancer: Young International Society of Geriatric Oncology review paper. J Geriatr Oncol. 2020; 11:217-24. https://doi.org/10.1016/j. jgo.2019.09.005

19. Chi C. The role of hope in patients with cancer. Oncol Nurs Forum 2017; 34:415-24. https://doi.org/10.1188/07.onf.415-424

20. Uslu-Sahan F, Terzioglu F, Koc G. Hopelessness, death anxiety and social support of hospitalized patients with gynecologic cancer and their caregivers. Cancer Nurs 2019; 1:1-8. https://doi.org/10.1097/ncc.0000000000000622

21. Tan M, Karabulutlu E. Social support and hopelessness in Turkish patients with cancer. Cancer Nurs 2005; 28:236-40. https://doi.org/10.1097/00002820-200505000-00013

22. Madani H, Pourmemari M, Moghimi M, Rashvand F. Hopelessness, perceived social support and their relationship in Iranian patients with cancer. Asia Pac J Oncol Nurs 2018; 5:314-9. https://doi.org/10.4103/apjon.apjon_5_18

23. Barrios AK. La investigación del apoyo social en pacientes con cancer. Actualización de su estudio en Latinoamérica en los últimos 10 años [Tesis de grado]. Santa Marta: Universidad Cooperativa de Colombia: 2018.

24. Hernández R, Fernández C, Baptista L. Metodología de la investigación. México: McGraw Hill; 2014

25. Zimet G, Dahlem N, Zimet S, Farley G. The Multidimensional Scale of Perceived Social Support. J Pers Assess 1988; 52:30-41. https://doi.org/10.1207/s15327752jpa5201_2

26. Landeta O, Calvete E. Adaptación y validación de la Escala Multidimensional de Apoyo Social Percibido. Ansiedad y Estrés 2002; 8:173-182

27. Juárez JA. Adaptación de la Escala Multidimensional de Apoyo Social Percibido en universitarios de San Juan de Lurigancho, 2018 [Tesis de grado]. Lima: Universidad César Vallejo: 2018.

28. Beck AT, Weissman A, Lester D, Trexler L. The measurement of pessimism: The Hopelessness scale. J Consult Clin Psychol 1974; 42:861-5. https://doi.org/10.1037/ h0037562 
29. Aliaga JR, Rodríguez L, Ponce C, Frisancho A, Enríquez J. Escala de Desesperanza de Beck (BHS): Adaptación y Características psicométricas. Revista IIPSI 2006; 9:69-79.

30. Pérez P, González A, Mieles I, Uribe AF. Relación del apoyo social, las estrategias de afrontamiento y los factores clínicos y sociodemográficos en pacientes oncológicos. Pensamiento Psicológico 2017; 15:41-54. https://doi.org/10.11144/Javerianacali.PPSI152.rase

31. Garcés MV. Ansiedad y desesperanza en pacientes oncológicos del Hospital Carlos Andrade [Tesis de grado]. Quito: Universidad Central del Ecuador: 2019

32. Medrano RR, Rodríguez CB. Nivel de incertidumbre y desesperanza aprendida en pacientes con cáncer de cuello uterino del IREN sur, Arequipa 2018 [Tesis de grado]. Arequipa: Universidad Nacional de San Agustín de Arequipa: 2018

33. Yildirim Y, Sertoz OO, Uyar M, Fadiloglu C, Ruchan U. Hopelessness in Turkish cancer inpatients: The relation of hopelessness with psychological and disease-related outcomes. Eur J Oncol Nurs 2009; 13:81-6. https://doi.org/10.1016/j.ejon.2009.01.001

34. Yagmur Y, Duman M. The relationship between the social support level perceived by patients with gynecologic cancer and mental adjustment to cancer. Int J Gynaecol Obstet 2016; 134:208-11. https://doi.org/10.1016/j.ijgo.2015.12.010

35. Kadambi S, Soto-Perez-de-Celis E, Garg T, Loh K, Krok-Schoen J, Battisti N, Moffat G, Gil-Jr L, Mohile S, Hsu T. Social support for older adults with cancer: Young International Society of Geriatric Oncology review paper. J Geriatr Oncol 2020; 11:217-224. https:// doi.org/10.1016/j.jgo.2019.09.005

36. Rengifo M. Apoyo Social percibido y recibido en mujeres diagnosticadas con cáncer de seno [Tesis de grado]. Cali: Pontificia Universidad Javeriana: 2017.

37. Sahin ZA, Tan M, Polat H. Hopelessness, depression and social support with end of life Turkish cancer patients. Asian Pacific J Cancer Prev 2013; 14:2823-2828. https://doi. org/10.7314/APJCP.2013.14.5.2823

38. Bayat M, Erdem E, Gül Kuzucu E. Depression, anxiety, hopelessness, and social support levels of the parents of children with cancer. J Pediatr Oncol Nurs 2008; 25:247-53. https://doi.org/10.1177/1043454208321139

39. Norskov K, Overgaard D, Lomborg K, Kjeldsen L, Jarden M. Patients' experiences and social support needs following the diagnosis and initial treatment of acute leukemia - A qualitative study. Eur J Oncol Nurs 2019; 41:49-55. https://doi.org/10.1016/j. ejon.2019.05.005

40. McNeil R, Egsdal M, Drew S, McCarthy M, Sawyer S. The changing nature of social support for adolescents and young adults with cancer. Eur J Oncol Nurs 2019; 43:1-7. https://doi.org/10.1016/j.ejon.2019.09.008

41. Abanto EP, Sachún LC. Funcionamiento familiar, apoyo social y resiliencia en el adulto oncológico. Hospital de alta complejidad Virgen de la puerta - Trujillo, 2016 [Tesis de grado]. Trujillo: Universidad Privada Antenor Orrego: 2016.

42. Galán S, de la Vega R, Tomé C, Racine M, Solé E, Jensen M, Miró J. What are the needs of adolescents and young adults after a cancer treatment? A Delphi study. Eur J Cancer Care 2017; 26:1-10. https://doi.org/10.1111/ecc.12488

43. Borneman T, Stahl C, Ferrell B, Smith D. The Concept of hope in family caregivers of cancer patients at home. J Hosp Palliat Nurs 2002; 4:21-33. https://doi.org/10.1097/00129191200201000-00012

44. Pousa V, Miguelez A, Hernández M, González M, Gaviria M. Depresión y Cáncer: Una revisión orientada a la práctica clínica. Rev Colomb Cancerol 2015; 19:166-172. https:// doi.org/10.1016/j.rccan.2015.04.005 
45. Beedie A, Kennedy P. Quality of social support predicts hopelessness and depression post spinal cord injury. J Clin Psychol Med Settings 2002; 9:227-34. https://doi. org/10.1023/A:1016003428370

46. Boinon D, Sultan S, Charles C, Stulz A, Guillemeau C, Delaloge S, Dauchy S. Changes in psychological adjustement over the course of treatment for breast cancer: the predictive role of social sharing and social support. Psychooncology 2013; 23:291-8. https://doi. org/10.1002/pon.3420

47. Holmegaard K, Overgaard D, Lomborg K, Kjeldsen L, Mary J. Patients' experiences and social support needs following the diagnosis and initial treatment of acute leukemia - A qualitative study. Eur J Oncol Nurs 2019; 34:49-55. https://doi.org/10.1016/j. ejon.2019.05.005

48. You J, Lu Q. Sources of social support and adjustment among Chinese cancer survivors: gender and age differences. Support Care Cancer 2014; 22:697-704. https://doi. org/10.1007/s00520-013-2024-z

49. Ferrell BR, Smith SL, Juarez G, Melancon C. Meaning of illness and spirituality in ovarian cancer survivors. Oncol Nurs Forum 2003; 30:249-58. https://doi.org/10.1188/03. ONF.249-257

50. Hill PC, Pargament KI. Advances in the conceptualization and measurement of religion and spirituality. implications for physical and mental health research. Am Psychol 2003; 58:64-74. https://doi.org/10.1037/0003-066x.58.1.64

51. Smith TB, McCullough ME, Poll J. Religiousness and Depression: Evidence for a Main Effect and the Moderating Influence of Stressful Life Events. Psycho Bull 2003; 129: 614-36. https://doi.org/10.1037/0033-2909.129.4.614

52. Benzein EG, Berg AC. The level of and relation between hope, hopelessness and fatigue in patients and family members in palliative care. Palliat Med 2005; 19:234-40. https:// doi.org/10.1191/0269216305pm1003oa

53. Okano Y, Okamura H, Watanabe T, Narabayashi M, Katsumata N, Ando M et al. . Mental adjustment to first recurrence and correlated factors in patient with breast cancer. Breast Cancer Res Treat 2001; 67:255-62. https://doi.org/10.1023/a:1017942709369

54. Lo C, Zimmermann C, Rydall A, Walsh A, Jones J, Moore M, Shepherd F, Gagliese L, Rodin G. Longitudinal study of depressive symptoms in patients with metastatic gastrointestinal and lung cancer. J Clin Oncol 2010; 28:3084-9. https://doi.org/10.1200/ JCO.2009.26.9712

55. Greihman G, Zimmermann C, Deckert A, Fitzgerald P, Mischitelle A, Rydall A et al.. Depression and hopelessness in patients with acute leukemia: the psychological impact of and acute and life-threatening disorder. Psychooncology 2016; 25:979-89. https://doi. org/10.1002/pon.3940 